\title{
Commentary
}

\section{Strategic Analysis of COVID-19 Response Measures and Psychological Intervention in China}

\author{
Ying $\mathrm{Wu}^{1}$, Bin Pan ${ }^{2, ~ *}$, Yutong $\mathrm{Li}^{2,}$, , Jinai He${ }^{1}$, Guohua Huang ${ }^{1}$, Qiufeng $\mathrm{Li}^{1}$ \\ ${ }^{1}$ Department of Nursing, The First Affiliated Hospital of Jinan University, Guangzhou, China \\ ${ }^{2}$ Department of Urology, The First Affiliated Hospital of Jinan University, Guangzhou, China
}

\section{Email address:}

wuying20050511@126.com (Ying Wu),panbin@jnu.edu.cn (Bin Pan), liyutong@jnu.edu.cn (Yutong Li), jinaihe@21cn.com (Jinai He), jndxhgh@163.com (Guohua Huang),gga.2000@163.com (Qiufeng Li)

${ }^{*}$ Corresponding author

\section{To cite this article:}

Ying Wu, Bin Pan, Yutong Li, Jinai He, Guohua Huang, Qiufeng Li. Strategic Analysis of COVID-19 Response Measures and Psychological Intervention in China. American Journal of Biomedical and Life Sciences. Vol. 8, No. 2, 2020, pp. 41-46. doi: 10.11648/j.ajbls.20200802.13

Received: March 31, 2020; Accepted: April 13, 2020; Published: April 23, 2020

\begin{abstract}
At the end of 2019, an ongoing outbreak of novel Corona Virus Disease 2019 (COVID-19) was first found in Wuhan, China with a sign of person-to-person transmission and city-to-city transmission, and has spread globally. At present, the COVID-19 mortality rate in China is $2.3 \%$, while the SARS was $9.6 \%$ and the MERS was $34.4 \%$, reported by WHO. China improved the surveillance network when the outbreak occurred and the labs promptly identified and shared the coronavirus genome that has served greatly to effective epidemic control. Given the fact that the number of infections in China is gradually declining while that outside of China keeps rising, more comprehensive information about the features, origin, epidemiology, clinical spectrum of COVID-19 is to be identified by further studies. The analysis of Chinese WHO-affirmed effective prevention and control strategies will help China and the rest of the world better contain this outbreak and end it as soon as possible. At present, effective prevention measures for COVID-19 include isolating the infectious agents, curbing the route of transmission, conducting health education to citizens, making fewer trips outside, washing hands frequently, wearing masks, protecting eyes, showing more understanding and consolation to patients, especially to elderly patients, providing mental support for medical staff and employing y psychological intervention timely.
\end{abstract}

Keywords: COVID-19, Symptom, Transmission, Quarantine, Health Education, Psychological Crisis Intervention

\section{Background}

Corona Virus Disease 2019 (COVID-19) was first found in Wuhan, China in the end of 2019, indicating person-to-person transmission and city-to-city transmission $[1,2]$. By February 12, 2020, 59.05 confirmed cases were identified in China along with 1,367 deaths, and 5,911 discharged cases. Later, after taking active prevention and control measures, by March 12, 2020, there were 13,526 confirmed cases under treatment in China, with total 64,111 cured and discharged cases, 80,813 confirmed cases, and 3,176 deaths (See Figure 1) [3]. The good news now is that the cured cases outnumber the newly-admitted cases in daily reports and many places outside Hubei province have achieved zero growth. Though China reacted quickly and soon identified the genome of coronavirus but still did not manage to stop it from spreading globally. By March 12, 2020, 47,919 confirmed cases and 1,574 deaths have reported in other 119 countries except China [3] which include 12,462 confirmed cases and 827 deaths in Italy, 9,000 confirmed cases and 354 deaths in Iran, 7,869 confirmed cases and 66 deaths in Korea, 2,281 confirmed cases and 48 deaths in France, 2,182 confirmed cases and 49 deaths in Spain, 1,567 confirmed cases in Germany, 1,162 confirmed cases and 37 deaths in the United States etc. (see Figure 2). WHO claimed that the epidemic may reach its peak around the world in the next several weeks [4], and declared this a health public emergency. Therefore, 
more knowledge of the virus's features, origin, epidemiology, clinical spectrum is to be fulfilled. Given the fact that infections in China are declining (see Figure 3), this article is designed to analyse China's effective prevention and control measures for COVID-19 to draw more valuable experience.

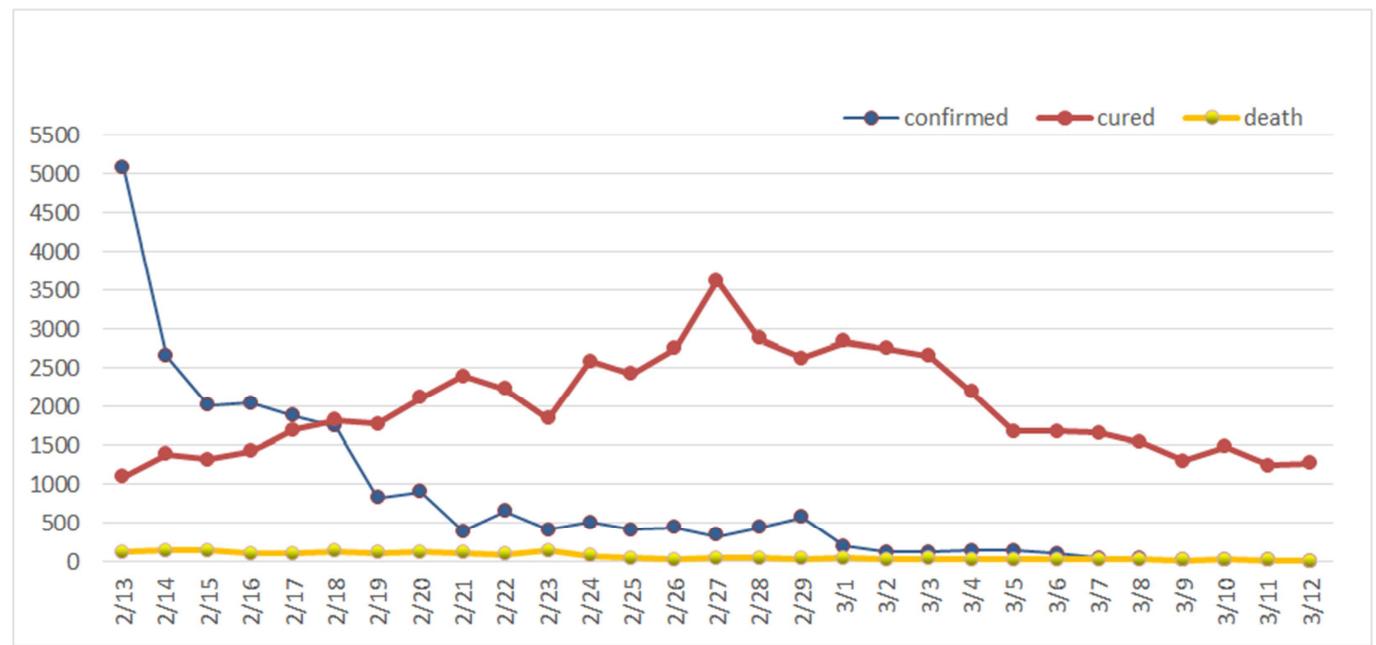

Source: National Health Commission of the People's Republic of China.

Figure 1. The number of new confirmed/ cured/death cases in China by Mar 12, 2020.

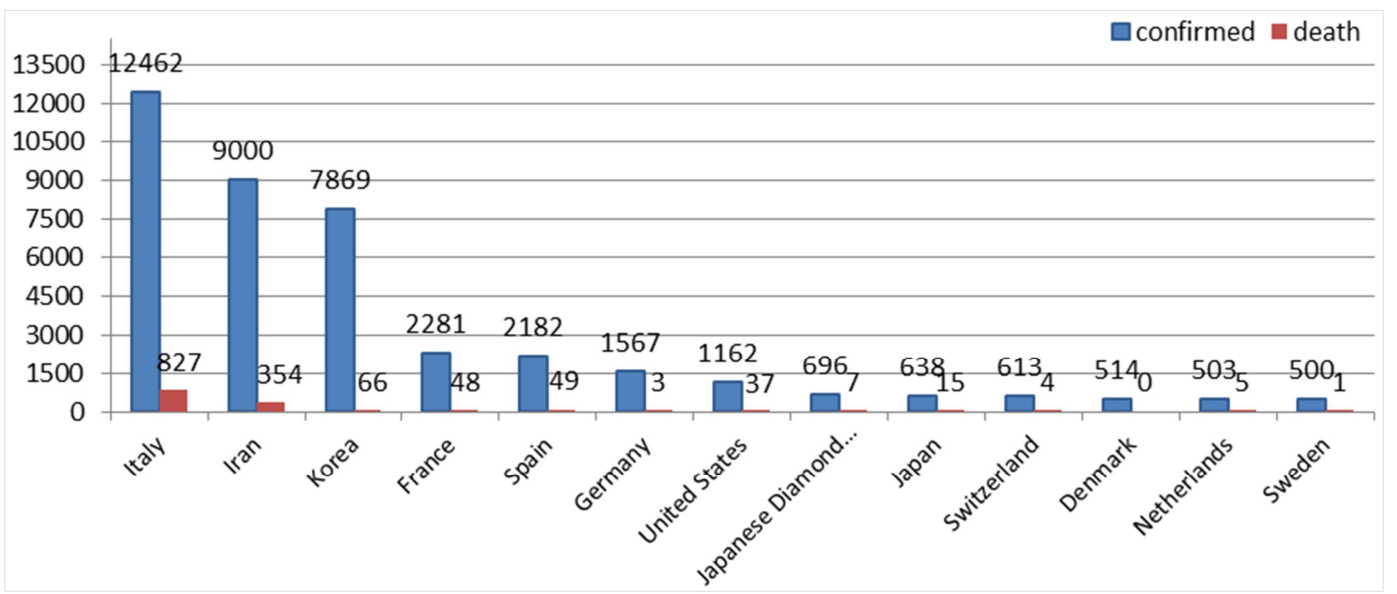

Source: World Health Organization.

Figure 2. The number of confirmed and death cases outside China by Mar 12, 2020.

\section{The number of confirmed cases in China}

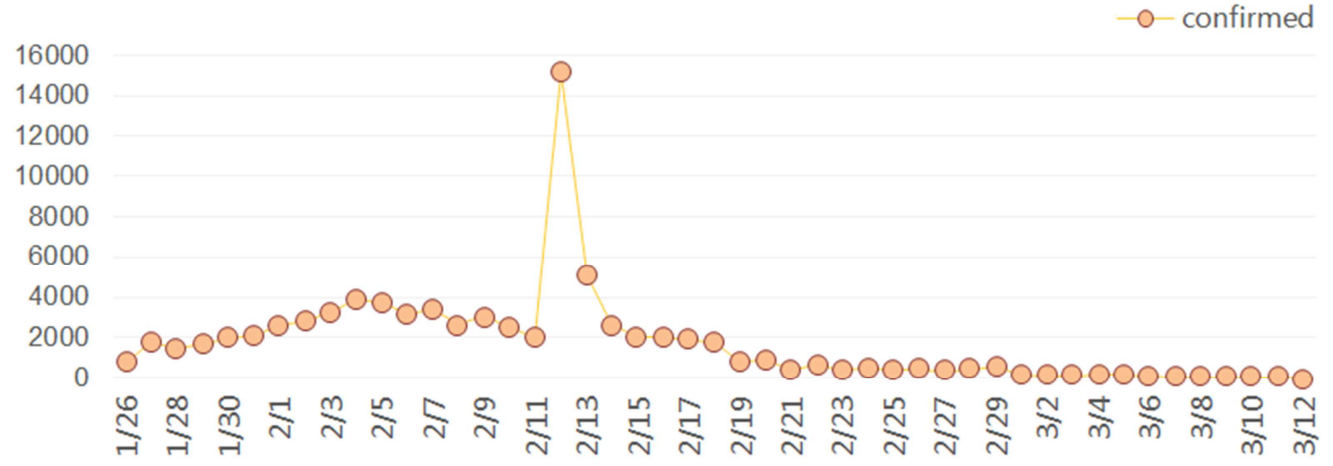

Source: National Health Commission of the People's Republic of China.

Figure 3. The number of new confirmed cases in China by Mar 12, 2020. 


\section{Epidemiological Features of 2019-nCoV}

Coronaviruses are a kind of enveloped single-stranded RNA virus that distribute broadly in humans, other mammals and birds, and can easily cause respiratory, intestinal, hepatic, and neurological diseases [5]. It belongs to the $\beta$-coronavirus and is polymorphic in the shape of round or oval, 60-140nm in diameter. Current studies indicate that it shares high homology (over 85\%) with bat-SL-CoVZC45, similar to SARS-CoV and MERS-CoV [5], but is significantly different in genetic features [6]. When cultured in vitro, the virus can be found in human respiratory epithelial cells in about 96 hours, and takes about 6 days when cultured in Vero E6 and Huh-7 cell lines. It is sensitive to ultraviolet light and heat (killed at 56 degree Celsius for 30 minutes), and lipid solvents like ether, $75 \%$ ethanol, chlorine-containing disinfectant, peracetic acid and chloroform which can effectively inactivate the virus except for chlorhexidine.

\subsection{Origin}

Most early-infected patients in China had connection (work in or live nearby) with the South China Seafood Wholesale Market in Wuhan which sells live wild animals. However, 13 of 41 patients had no connection with the market [7]. Most importantly, one of the earliest cases, found in Dec 12, 2019, had no exposure to the seafood market. Therefore, the origin of COVID-19 is to be determined.

\subsection{Infectious Agents}

According to a report from Chinese Center for Disease Control and Prevention (China CDC), laboratory tests have ruled out SARS-CoV, MERS-CoV, influenza, avian influenza, adenovirus and other common respiratory pathogens, and considered the 2019-nCoV as the pathogen causing the outbreak. The increasing number of cases evidenced that the 2019-nCoV is transmissible among humans, and even asymptomatic carriers can be a source of infection.

\subsection{Route of Transmission}

Main routes of transmission include droplet transmission and close contact transmission, and possible aerosol transmission in a relatively closed environment for a long time exposure to high concentrations of aerosol.

\subsection{Susceptible Population}

A large number of cases have proved that the population is generally susceptible. The $2019-\mathrm{nCoV}$ onsets in a cluster of crowd, and is more likely to infect old patients with comorbidities and even results in fatal respiratory diseases [11]. At present, the mortality of $2019-\mathrm{nCoV}$ in China is $2.3 \%$ [12], compared with $9.6 \%$ of SARS and $34.4 \%$ of MERS reported by WHO $[13,14]$. Based on the current data, this virus may not be as fatal as the public assume.

\section{Clinical Spectrum}

\subsection{Symptoms}

According to current epidemiological researches, the incubation period is generally 3-7 days (within 14 days), mainly featured with fever, dry cough and fatigue. Few patients may accompany with symptoms like rhinobyon, rhinorrhea, pharyngalgia, myalgia and diarrhea, which are similiar to the onset of SARS and MERS. Shortness of breath or hyoxemia after a week of the illness onset in sever patients and might develop into acute respiratory distress syndrome (ARDS), followed by septic shock, refractory metabolic acidosis, coagulation dysfunction and multiple organ failure, if the disease could not be controlled in time. Notably, sever and critical patients may have low fever or even no significant fever, and most mild patients can fully recover and children have relatively light symptoms $[16,17]$ with less favorable prognosis for the elderly and those with chronic diseases. The proportion of mild, severe and critical patients is about $80 \%, 13 \%$, and $6 \%$, respectively [15].

\subsection{Laboratory Findings}

In the early stages of the disease, researchers found normal or decreased number of white blood cells in the peripheral blood and decreased lymphocyte count in general cases, and increased liver enzymes, lactic dehydrogenase (LDH), creatase, and myoglobin in some cases, and increased troponin in some critical cases. Most patients have elevated C-reactive protein (CRP) and erythrocyte sedimentation rate and normal procalcitonin. Sever patients usually have increased D-dimer and progressively decreased peripheral blood lymphocytes, and critical patients usually accompany with elevated inflammatory factors. Also, novel coronavirus nucleic acids can be detected in nasopharyngeal swabs, sputum and other lower respiratory tract secretions, blood, and feces [2, 17]. In order to improve the positive rate of nucleic acid detection, it is recommended to collect sputum or lower respiratory tract secretions from trachea-intubated patients and then conduct tests as soon as possible.

\subsection{Imageology Findings}

Early chest CT scans of infected patients show multiple lobular and interstitial changes, apparently in peripheral lung areas, and later develop into multifocal ground-glass changes which are typical features of viral pneumonia, and bilateral ground-glass opacity and infiltrates, or even lead to consolidation of the lungs but not commonly accompany with pleural effusions. The ground-glass changes showed in chest CT scans appear earlier than the positive results of RT-PCR [2, 17]. 


\section{Prevention and Management Strategies}

The three basic elements of infectious epidemic are the infectious agents, the route of transmission and the susceptible population so eliminating the source of infection and blocking the route of transmission are usually considered to be effective approaches to stop the spread of infectious diseases. More and more cases prove that COVID-19 is highly transmissible from person to person, and super-spreading events have led to large-scale outbreaks in public assembly.

\subsection{Management on the Source of Infection}

In order to control the spread of COVID-19, Chinese authorities have set up pre-screening spots at medical institutions at all levels to prevent cross-infection within the institutions, and personnel who work there are given primary protection. All patients in outpatient clinic, emergency department rtment and inpatients shall be performed pre-screening and triage, asked one by one for their historical epidemiology, fever, or respiratory symptoms, and get registered. Later, the patients $t$ will be given a mask and sent to the corresponding general department for treatment after they are ruled out of infection. Physicians in each department shall ask the patients about their historical epidemiology and do screening for infectious diseases again based on the their main complaint, medical history, symptoms and signs. Medical institutions shall also set up standard fever clinics, complete secondary protection and improve the isolation areas to receive febrile patients. Also, standard isolation wards shall be set up for isolating COVID-19 suspected patients who should be reported to the health department of the jurisdiction, and transferred to designated hospitals for further examination and treatment according to the guidelines COVID-19 confirmed patients shall be isolated and treated at a nearby designated hospital in a centralized manner and managed according to their conditions (mild, normal, sever, critical). Medical staff there shall be provided three-level protection. Experts are organized to formulate a standardized diagnosis and treatment plan, and closely monitor the changes in patients' condition, and more importantly, have timely diagnosis for patients with advanced age, obesity, pregnancy, comorbidities, and rapid progression so as to prevent exacerbation. Patients who meet the COVID-19 discharge standard can be discharged and are recommended to continue to stay at home for 14 days, take less trips outside and avoid going to crowded places.

The huge demand for the COVID-19 outbreak has challenged the health workers and the national medical supply system [18]. New cases surged in the early stage of COVID-19 emergencies, and the Chinese authorities took quick actions to well prepare supplies, build the Leishenshan Hospital and Huoshenshan Hospital in Wuhan at top speed in 10 days, with a total of 2,000 beds, and then continued to build 16 temporary treatment centers for infected patients. So far, the number of hospital beds in Wuhan has increased from over 5,000 before the epidemic to 23,000 , including 9,000 intensive care beds. At the same time, more than 40,000 medical staff across the country volunteered to assist in Wuhan. There has been more than 500 doctors and nurses from The First Affiliated Hospital of Jinan University volunteering to assist in Wuhan with 89 have been engaging in the treatment of COVID-19 critical patients there. Now, the Chinese government has managed to speed up the diagnosis for fever patients by prompt nucleic acid testing and chest $\mathrm{CT}$ examinations so as to make sure suspected patients can be admitted, tested and diagnosed in a short time and receive timely treatment with the cost of treatment for all COVID-19 patients nationwide funded by the government as well. Since February 13, 2020, the number of new confirmed cases in Wuhan has started to decrease significantly while that of cured cases has continued to increase (see Figure 1).

Also, The Chinese government has strengthened the training of COVID-19 in medical institutions at all levels, introducing its epidemiological and clinical features, and diagnosis and treatment plans to raise the capabilities to deal with the outbreak. Professional infection-control supervisors have been dispatched to hospitals to monitor and correct non-standard behaviors in time, and reduce the risk of exposure to infection for medical staff as well as their metal stress [19].

\subsection{Management on the Route of Transmission}

In order to cut off the transmission route, the Chinese authorities have set up screening spots to monitor all personnel at the border of cities and towns, and all the entrances and exits of highways, coach stations, railway stations, terminals, airports, residences, hotels, etc., and complete a comprehensive registration and management system for febrile patients. Any discovered fever patients there would be taken in by the designated medical institutions in each region. For those who have close contact with COVID-19 patients and come from Wuhan, a 14-day quarantine is requested and health monitoring will be performed on daily basis, and they shall seek for medical help immediately once symptoms like fever and cough occur. One of the greatest move for Chinese government was implementing a lockdown in Wuhan, center of the affected areas, which successfully obtained the window period of COVID-19 infections, and allowed neighboring cities and other countries around the world to have more time and opportunities to prepare for the epidemic.

\subsection{Psychological Intervention}

COVID-19, as a public health emergency, is highly likely to trigger people's concern and panic so it is necessary to timely assess the mental health of infection-stricken population so as to provide mental health services and psychological crisis intervention as soon as possible to reduce and eliminate the psychological damage caused by the epidemic, and try to minimum its negative psychological impact to the society. 


\subsubsection{Psychological Interventions for COVID-19 Patients During Treatment}

Infected patients during quarantine and treatment usually show denial, anger, fear, anxiety, depression, disappointment, complaints, insomnia, loneliness, and even aggressiveness, and may be uncooperative or withdraw from treatment due to fear of the disease, or be overly optimistic and have high expectation of the treatment, which are all normal stress responses and should be understood. Therefore, it is important to evaluate patients' mental health in a timely manner, explain the importance and necessity of isolation and treatment, and encourage them to build a positive attitudes towards recovery. Also, they shall be assisted in communicating with their loved ones to exchange information and consult a psychiatrist during treatment when necessary.

\subsubsection{Psychological Interventions for Suspected Patients During Quarantine}

Suspected patients during quarantine usually show panic, restlessness, loneliness, helplessness, depression, pessimism, anger, tension, fluke, avoidance of treatment, pressure to be alienated from others, grievance, shame, or disregard for illness etc.. To ease these situations, patients should take in scientific, reliable and authoritative source or knowledge, be encouraged to actively cooperate with treatment and isolation, have healthy eating and good rest, read more, listen to music, have more communication with others and resume daily activities by modern telecommunication technologies, understand and accept current isolation and their own responses, and be positive. Also, they are encouraged to seek help from psychic hotline or engage in online psychological intervention when necessary.

\subsubsection{Psychological Interventions for Old People}

A large number of COVID-19 death cases involve old people [9] so it is extremely significant to provide more help, comfort and support for them in this special period [21]. The rapid spread and high mortality of severe acute respiratory syndrome coronavirus (SARS-CoV-2) may exacerbate the risk of mental health problems and existing mental symptoms, further impairing their daily functions and cognition. Although online mental health services have been widely adopted [20], old people only have limited access to Internet and smartphones so only a small percentage of them can benefit from such services.

\subsubsection{Psychological Interventionss for Medical Personnel}

Medical personnel is facing tremendous physical and mental stress in the face of this large-scale infectious public health emergency [22]. For employees, their first concern is not about being infected but making their families worried or even endangering their health if accidentally bring the virus home. Also, they have to deal with some patients who are reluctant to accept isolation and treatment due to the fear or ignorance of the diseases. With the large-scale outbreaks of infectious diseases, they can easily become overly fatigued, nervous, and even exhausted, or suffer from anxiety, insomnia, depression, sadness, grievance, frustration or self-blame in the face of patient death. They may also be excessively excited, refusal of reasonable rest, and neglect their personal health. Therefore, it is necessary for them to take psychological crisis intervention training before participating in rescue, understand the stress responses and learn how to cope with that to regulate their emotions. Also, they can take part in preventive interviews to openly discuss inner feelings, give each other support and comfort, and mobilize resources so as to have better psychological preparation for their work and to eventually allay the worries of front-line medical workers [23].

\subsection{Health Education}

The Chinese authorities have widely publicized the information and knowledge of COVID-19, including common symptoms and protective measures (washing hands frequently, wearing masks, preventing from rubbing eyes with your hands, disinfecting and keeping ventilation indoors, taking few trips outside, staying away from the crowd, and seeking medical help immediately if symptoms occur) to schools, enterprises, communities and public places etc. through news, newspapers, Internet, broadcast in towns and villages and other channels. In hospitals, medical workers also introduce that to patients through distributing leaflets and exchanging with them. In this case, almost every Chinese people knows how to prevent COVID-19 by approaches like disposing used masks and garbage properly and not eating wild animals. Some Chinese people used to consider wild animals like pangolins and bats as natural, pollution-free and nutrition-rich food that can enhance immunity but now the COVID-19 epidemic has taught people a profound lesson that enacting strict laws on banning people from eating wild animals is an urgent.

\section{Conclusion}

The epidemic of COVID-19 breaks out in China and globally from the end of 2019 to the beginning of 2020. Researches show that the genome sequences of $2019-\mathrm{nCoV}$ are highly identical (over 85\%) to the bat-SL-CoVZC45, similar to SARS-CoV and MERS-CoV. At present, the mortality of COVID-19 in China is $2.3 \%$, while the SARS was $9.6 \%$ and the MERS was $34.4 \%$, reported by WHO. The proportion of mild, severe and critical patients is about $80 \%, 13 \%$, and $6 \%$, respectively. Common symptoms of COVID-19 include fever, dry cough and fatigue, and few patients may accompany with rhinobyon, rhinorrhea, pharyngalgia, myalgia and diarrhea etc.. Sever patients usually suffer from shortness of breath or hyoxemia after a week of the illness onset and might develop into acute respiratory distress syndrome (ARDS), if the disease could not be controlled timely. The ground-glass changes showed in chest CT scans appear earlier than the positive results of RT-PCR. China now has achieved remarkable in COVID-19 prevention. Report of the WHO-China joint mission on COVID-19 states that China has adopted the bravest, most flexible and proactive prevention and control measures in history [24]. It manages to effectively curb the rapid spread of this new respiratory pathogen, reverse the escalating process of rapidly spreading infections, showing more 
understanding and consolation to patients, especially to elderly patients, providing mental support for medical staff and employing y psychological intervention timely, and provide vital experience for the global response to this novel and striking COVID-19. Hope these experience can help China and the rest of the world better respond to the current outbreak as well as to the future outbreaks of unexpected infectious diseases.

\section{Acknowledgements}

We thank Zhenshu Wu for assistance COVID-19 in Wuhan, China: Effect Analysis of Prevention and Control Strategies.

\section{References}

[1] Zhu N, Zhang D, Wang W, Li X, Yang B, Song J, et al. A Novel Coronavirus from Patients with Pneumonia in China, 2019. N Engl J Med. 2020, 382 (8): 727-733.

[2] Huang CL, Wang YM, Li XW, Ren L, Zhao J, Hu Y, et al. Clinical features of patients infected with 2019 novel coronavirus in Wuhan, China. Lancet. 2020, 395 (10223): 497-506.

[3] Liu K, Fang YY, Deng Y, Liu W, Wang MF, Ma JP, et al. Clinical characteristics of novel coronavirus cases in tertiary hospitals in Hubei Province. Chin Med J (Engl). 2020 Feb 7. doi: 10.1097/CM9.0000000000000744. [Epub ahead of print].

[4] Interim infection prevention and control recommendations for patients with known or patients under investigation for 2019 novel coronavirus $(2019-\mathrm{nCoV})$ in a healthcare setting. https://www.cdc.gov/coron avirus/2019-nCoV/hcp/infec tion-contr ol.html. Accessed 30 Jan 2020.

[5] Weiss SR, Leibowitz JL. Coronavirus pathogenesis. Adv Virus Res. 2011, 81: 85-64.

[6] Song Z, Xu Y, Bao L, Zhang L, Yu P, Qu Y, et al. From SARS to MERS, thrusting coronaviruses into the spotlight. Viruses. 2019; 11 (1) pii: E59.

[7] Chen N, Zhou M, Dong X, Qu J, Gong F, Han Y, et al. Epidemiological and clinical characteristics of 99 cases of 2019 novel coronavirus pneumonia in Wuhan, China: a descriptive study. Lancet. 2020, 395 (10223): 507-513.

[8] She J, Jiang J, Ye L, Hu L, Bai C, Song Y. 2019 novel coronavirus of pneumonia in Wuhan, China: emerging attack and management strategies. Clin Transl Med. 2020, 9 (1): 19.

[9] Li Q, Guan XH, Wu P, Wang X, Zhou L, Tong Y, et al. Early transmission dynamics in Wuhan, China, of novel coronavirus infected pneumonia. N Engl J Med. 2020 Jan 29. doi: 10.1056/NEJMoa2001316. [Epub ahead of print].

[10] Phan LT, Nguyen TV, Luong QC, Nguyen TV, Nguyen HT, Le HQ, et al. Importati on and human-to human transmission of a novel coronavirus in Vietnam. N Engl J Med. 2020, 382 (9): 872-874.

[11] Wang H, Wang Z, Dong Y, Chang R, Xu C, Yu X, et al. Phase-adjusted estimation of the number of Coronavirus Disease 2019 cases in Wuhan, China. Cell Discov. 2020, 6: 10.
[12] New features of novel coronavirus (2019-nCoV) pneumonia. https://new. qq.com/omn/20200128/20200128A07H4S00.html. Accessed 30 Jan 2020.

[13] Munster VJ, Koopmans M, van Doremalen N, van Riel D, de Wit E. A Novel Coronavirus Emerging in China-Key Questions for Impact Assessment. N Engl J Med. 2020, 382 (8): 692-694.

[14] WHO statement regarding cluster of pneumonia cases in Wuhan, China. https://www.who.int/china/news/detail/0901-2020-who-state.

[15] Chen ZL, Zhang Q, Lu Y, Guo ZM, Zhang X, Zhang WJ, et al. Distribution of the COVID-19 epidemic and correlation with population emigration from wuhan, China. Chin Med J (Engl). 2020 Feb 28. doi: 10.1097/CM9.0000000000000782. [Epub ahead of print].

[16] Wu JT, Leung K, Leung GM. Nowcasting and forecasting the potential domestic and international spread of the 2019-nCoV outbreak originating in Wuhan, China: a modelling study. Lancet. 2020, 395 (10225): 689-697.

[17] Chan JF, Yuan S, Kok KH, To KK, Chu H, Yang J et al. A familial cluster of pneumonia associated with the 2019 novel coronavirus indicating person-to-person transmission: a study of a family cluster. Lancet. 2020, 395 (10223): 514-523.

[18] Cao Y, Li Q, Chen J, Guo X, Miao C, Yang H, Chen Z, Li C. Hospital Emergency Management Plan During the COVID-19 Epidemic. Acad Emerg Med. 2020, Mar 2. doi: 10.1111/acem.13951.[Epub ahead of print].

[19] Chen Q, Liang M, Li Y, Guo J, Fei D, Wang L, He L, Sheng C, Cai Y, Li X, Wang J, Zhang Z. Mental health care for medical staff in China during the COVID-19 outbreak. Lancet Psychiatry. 2020, 18pii: S2215-0366 (20) 30078-X.

[20] Peeri NC, Shrestha N, Rahman MS, Zaki R, Tan Z, Bibi S, et al. The SARS, MERS and novel coronavirus (COVID-19) epidemics, the newest and biggest global health threats: what lessons have we learned? Int J Epidemiol. 2020, 22. pii: dyaa033. doi: 10.1093/ije/dyaa033. [Epub ahead of print].

[21] Yang Y, Li W, Zhang Q, Zhang L, Cheung T, Xiang YT. Mental health services for older adults in China during the COVID-19 outbreak. Lancet Psychiatry. 2020, 18, pii: S2215-0366 (20) 30079-1. doi: 10.1016/S2215-0366(20)30079-1. [Epub ahead of print].

[22] Wu P, Fang Y, Guan Z, Fan B, Kong J, Yao Z, et al. The psychological impact of the SARS epidemic on hospital employees in China: exposure, risk perception, and altruistic acceptance of risk. Can J Psychiatry 2009; 54 (5): 302-11.

[23] Lai CC, Shih TP, Ko WC, Tang HJ, Hsueh PR. Severe acute respiratory syndrome coronavirus 2 (SARS-CoV-2) and coronavirus disease-2019 (COVID-19): The epidemic and the challenges.Int J Antimicrob Agents. 2020 Feb 17: 105924. doi: 10.1016/j.ijantimicag.2020.105924. [Epub ahead of print].

[24] National Health Commission of the People's Republic of China. Diagnosis and treatment of new coronavirus pneumonia (version 5) http://www.nhc.gov.cn/. Accessed 30 Jan 2020. 